\title{
Design and Optimisation of a Slat Conveyor for Airport Application
}

\author{
Vatsal Singh, Sanskar Joshi, Sahil Shaikh, Siddheshwar Wakude, Dilip Panchal
}

\begin{abstract}
This manuscript deals with the design, analysis and optimization of a Slat Conveyor for bag handling at the Airports. The requirement here is to transport the bags from loading station to the unloading station which covers the distance of 28 metres. The specification provided are the approximate weight of each bag, the total weight to be transported between the stations and the height upto which it is transported. The Input parameters are reference to the design calculations. Proper material selection is done using appropriate standards like the ASME, CEMA and Ashby standard. With the proposed conveyor system the weight of the base frame will be reduced and the fatigue strength/cycle of drive shaft will be increased using the appropriate materials.
\end{abstract}

Keywords : Factor of Safey, Load, Shaft, Slat Conveyor.

\section{INTRODUCTION}

A conveyor is a mechanical device which helps in transporting heavy and bulky materials from one place to another. It has several components like slat, chain, bearings etc. Conveyors are usually driven using the motor. The type of conveyor system depends on the type of requirement of the industry. Some of its types are - Chain conveyor, roller conveyor, slat conveyor, gravity conveyor, belt conveyor. They are in so much use because of the various advantages that they provide The project is design and development of slat conveyor for the application of transporting bags at the airport. Conveyor mainly consists of components like sprocket, chain, drive assembly, take up assembly, electric motor, etc. Number of factors are included in the design and development of the conveyor like load calculation, conveyor chain selection, design of the various component, layout design, drafting, modelling, Finite element analysis, iterations on the design, etc.

The essential requirements of a good material handling system may be summarized as:

1) Efficient and safe movement of materials to the desired place.

Revised Manuscript Received on September 20, 2020.

* Correspondence Author

Vatsal Singh*, Final Year, Mechanical Engineering, MIT Academy of Engineering, Pune, India, Email: vatsalsingh@mitaoe.ac.in

Sanskar Joshi, Final Year, Mechanical Engineering, MIT Academy of Engineering, Pune, India. Email: srjoshi@mitaoe.ac.in

Sahil Shaikh, Final Year, Mechanical Engineering, MIT Academy of Engineering, Pune, India. Email: sashaikh@mitaoe.ac.in

Siddheshwar Wakude, Final Year, Mechanical Engineering, MIT Academy of Engineering, Pune, India. Email: srwakude@mitaoe.ac.in

Dilip Panchal, Associate Professor, MIT Academy of Engineering, Pune, India.

(C) The Authors. Published by Blue Eyes Intelligence Engineering and Sciences Publication (BEIESP). This is an open access article under the CC BY-NC-ND license (http://creativecommons.org/licenses/by-nc-nd/4.0/)
2) Timely movement of material when needed.

3) Supply of material at desired rate.

4) Storage of materials using minimum space.

\section{Problem statement}

Most of the times the materials used for making the shaft for various applications are either low carbon steel or medium carbon steel. The disadvantage with these materials is that they tend to undergo torsion and break whenever they are overloaded. So our purpose is to find the right material for the shaft.

\section{SHAFT}

Itcan undergo failure due to various factors. These include corrosion, wear, fatigue and overloading. The machine shafts rarely fail because of the wear and corrosion. The reasons are mainly fatigue or overloading and fatigue is most common between the two. The shafts undergo the fatigue failure if the same load is applied to it over a cycle of period, whereas in overloading the shaft breaks just after the high load is applied. Our objective here is to increase the fatigue strength per cycle of the shaft.

\section{BASE FRAME}

The slat conveyor has to carry the load of 12 Metric tonnes per hour at a height of 94 inches (approx.) inclined at an angle of $20^{\circ}$. Here we have tried to optimize the critical parts (shaft, baseframe) . By doing so a considerate amount of material is also saved.

\section{Literature Survey}

Makoto Kanehira gave us the idea about how different variety of chains can be used for power transmission, depending the type of application.

H.G Rachner tells us about the design of chains and its lubrication and also the factor of safety mostly preferred.

Ashveer Singh's paper tells us about the comparative outcomes after designing different components of conveyor based on different requirements

Huanyu Zhao discovered that the tension in the chain link of an excavator was measured by assessing the values of horizontal straight, pivot steering and differential steering .

Design of Conveyor chain link:- Chain manufactures specify the chain in their product range by breaking load. Some have quoted average breaking loads, some have quoted minimum breaking loads depending upon their level of confidence in their product. To obtain a design working load is necessary to apply a "factor of safety" to the braking load and this is an area where confusion has arisen.

Daniel J Fonseca, Gopal Uppal, Timothy J Greene explained how complex it becomes to select the components of the equipment. CEMA standards if followed in a right way makes it easier for human experts to take an unbiased decision .

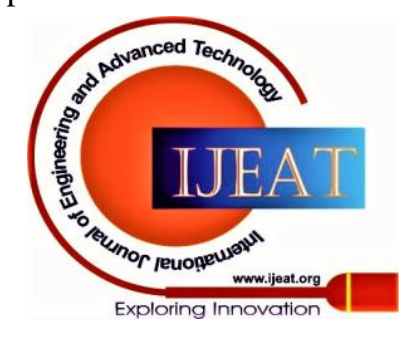




\section{Design and Optimisation of a Slat Conveyor for Airport Application}

Suitability score matters the most for fulfilling the need of the material handling conveyor.

\section{METHODOLOGY}

\section{A. Layout}

The layout of the Slat Conveyor gave us a brief idea about the components and their positions inside the conveyor.

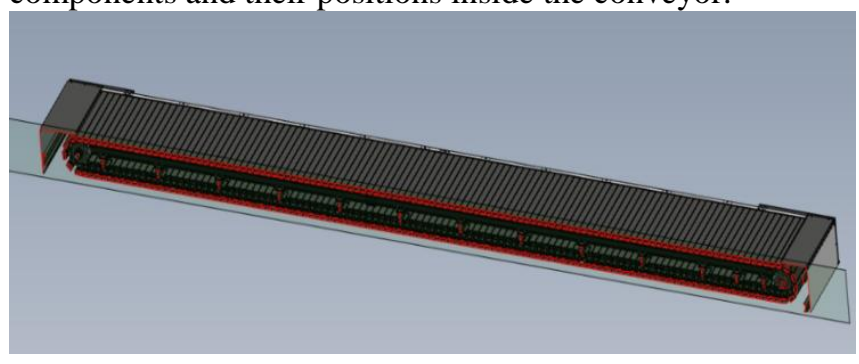

Fig 1: Isometric layout

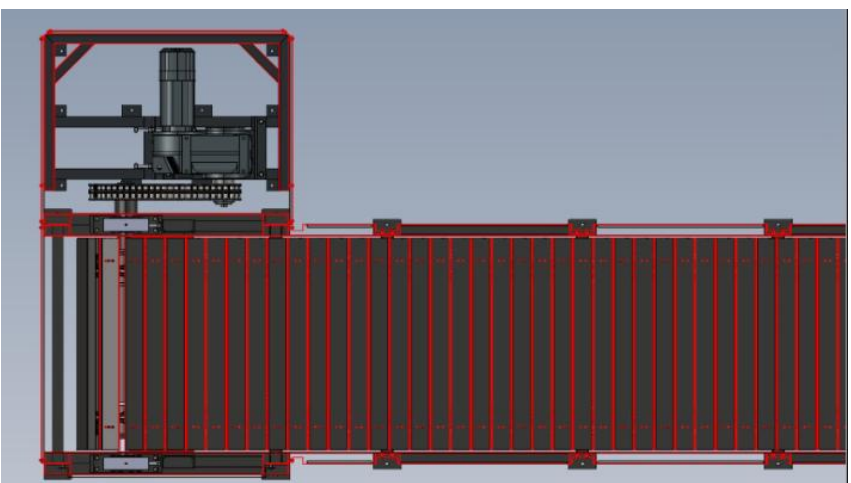

Fig 2: Top View

\section{B. Standards for Material Selection}

ASHBY STANDARD: Named after Michael Ashby, Ashby charts or Ashby plots are used for material selection. They are used to compare the ratio of properties of different materials. This graph helps us to recognize the material with the highest stiffness as well as the lowest density by using a log scale.

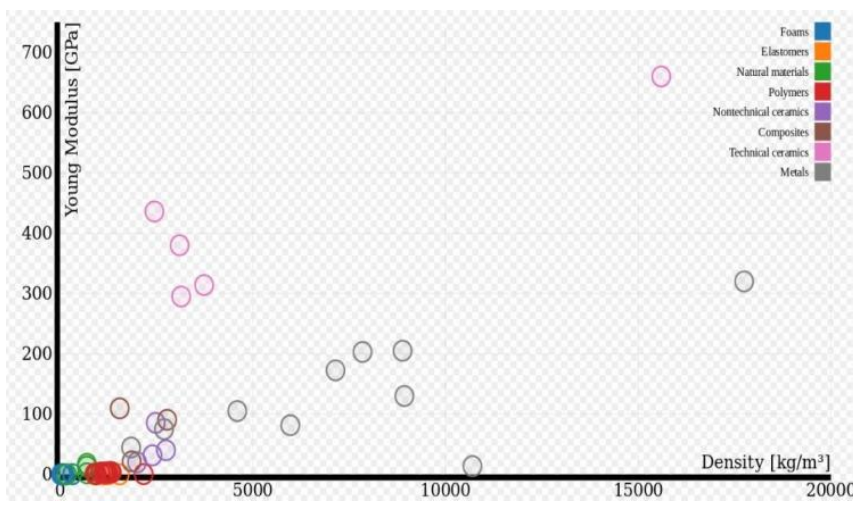

Cema Standard: Cema Or Conveyor Equipment Manufacturing Association Standard: CEMA is the standards that the companies usually follow while designing and manufacturing conveyors.

\section{Calculations}

Approach/Techniques applied:

Customer inputs

Material to be handled : Fertiliser Bags

Bag Size : 240mm*320mm*210 mm.

Bag Weight : Max. $=50 \mathrm{~kg}$, Min. $=28 \mathrm{~kg}$

Bag arrangement in cartons : Min.: 16 bags $(4 * 4)$ or Max.: 25 bags $(5 * 5)$.

Conveyor Length $=28 \mathrm{~m}$.
Conveyor Angle $=20^{\circ}$ (Inclined)

No. of Bags transported in 1 hour $=240$.

Bag handling rate (in mtph): $(240 * 50) / 1000=12 \mathrm{mtph}$.

Suggested Conveyor Speed according to CEMA Standards $: 0.25 \mathrm{~m} / \mathrm{s}$.

Speed in $\mathrm{m} / \mathrm{h}=900 \mathrm{~m} / \mathrm{h}$.

Slat Calculation

For Slat selection various section sizes are provided by the customer.

$\begin{array}{lll}75 * 40 * 3 & 100 * 75 * 5 & 125 * 75 * 6 \\ 75 * 40 * 5 & 100 * 75 * 6 & 125 * 75 * 8 \\ 75 * 40 * 8 & 100 * 75 * 8 & \end{array}$

Considering the Section Size $75 * 40 * 3$.

Material Used : IS2062. $(\mathrm{E}=210000 \mathrm{MPa}, \mathrm{Syt}=490 \mathrm{MPa})$.

Given Data:

Length $=800 \mathrm{~mm}$.

Weight of Each Carton (Considering Maximum Load) = $50 * 25=1250 \mathrm{~kg}=12262.5 \mathrm{~N}$

Desired FOS $=2$

Allowable Stress $=$ Syt $/ 2=490 / 2=245 \mathrm{MPa}$.

Max. Bending Moment $=\left(\mathrm{w}^{*} \mathrm{l}\right) / 4=(12252.5 * 0.8) / 4=$

2452.6N-m

Calculating the Moment of Inertia along $\mathrm{X}$ axis i.e.

$\mathrm{I}_{\mathrm{XX}}=39.29 * 10^{-8} \mathrm{~m}^{4}$.

Max. Bending Stress $=($ Maximum Bending Moment* y $) / \mathrm{I}$

(Moment of Inertia)

$=(2452.5 * 0.0375) /\left(39.29 * 10^{-8}\right)$

$=234076737.1 \mathrm{~N} / \mathrm{m}^{2}=234.076 \mathrm{~N} / \mathrm{mm}^{2}$.

Now the actual FOS = Allowable Stress $/$ Max. Bending Stress

FOS $=245 / 234.076=1.046$.

$(\text { FOS })_{\text {Actual }}<(\text { FOS })_{\text {Desired }}$, Hence the Design fails.

Similarly for Different Sections, by following the same above procedure, the FOS values found out below:

\begin{tabular}{|c|c|c|}
\hline $\begin{array}{c}\text { Section } \\
\text { Size }\end{array}$ & $\mathrm{I}_{\mathrm{XX}}\left(\mathrm{m}^{4}\right)$ & $\mathrm{FOS}_{\text {(Actual) }}$ \\
\hline $75 * 40 * 5$ & $60.5 * 10^{-8}$ & 1.6 \\
\hline $75 * 40 * 6$ & $69.8 * 10^{-8}$ & 1.85 \\
\hline $75 * 40 * 8$ & $86.2 * 10^{-8}$ & $\mathbf{2 . 2 9}$ \\
\hline
\end{tabular}

Since the section $75 * 40 * 8$ gives us the value of $\mathbf{2 . 2 9}$ as FOS which is greater than the Desired FOS.

Hence $\mathbf{7 5} \boldsymbol{*} \mathbf{4 0} \boldsymbol{*} \mathbf{8}$ can be selected for the slat design.

Development of Slat for section $75 * 40 * 8=$ 34+34+18.84+9.42+63 =161mm.

Mass of one slat $=(161 * 800 * 8 * 7.8) / 10^{6}=8.03 \mathrm{~kg} / \mathrm{m}$.

Chain Calculations:

Chain selected : SS911.

Pitch: 9 inches (225mm).

Allowable Pull Force $=4600$ lbs (2090 kgf).

Breaking Load = 29000 lbs (13181 kgf).

Weight of single Strand of chain $=12.7 \mathrm{lbs} / \mathrm{ft}$.

Weight of strand in S.I. unit $=12.7 * 1.5=19.05 \mathrm{~kg} / \mathrm{m}$.

Pin Diameter $=5 / 8$ inches $(16 \mathrm{~mm})$.

Roller Size $=3$ inches $(75 \mathrm{~mm})$.

Roller rolling friction coefficient $=(0.6 *$ Pin diameter $)$

Roller Diameter

$=(0.6 * 16) / 75=0.128$.

Double Strand weight = $19.05 * 2=38.1 \mathrm{~kg} / \mathrm{m}$.

Published By:

Blue Eyes Intelligence Engineering and Sciences Publication

(C) Copyright: All rights reserved.

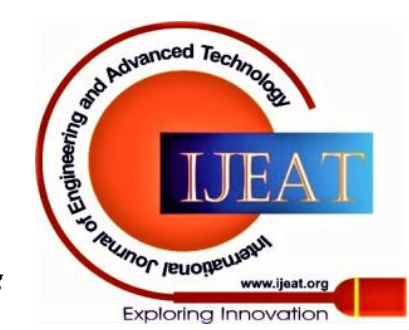


Slat weight with double strand weight $=38.1+8.03=46.13$ $\mathrm{kg} / \mathrm{m}$.

Weight of one slat $=8.03 \mathrm{~kg} / \mathrm{m}$.

No. of slats per metre $=1000 / 75=13.33=14$ slats

No. of slats for $28 \mathrm{~m}=28 * 14=392$ slats

Weight carried by the conveyor for 1 metre $=1250 * 14=$ $17500 \mathrm{~kg} / \mathrm{m}$.

Total weight carried per metre $=17500+(8.03 * 14)+38.1=$ $17650.52 \mathrm{~kg} / \mathrm{m}$

Carrying Run Resistance $=$ length of Conveyor*Total

weight* $\cos \varnothing * 0.15$

$=28 * 17650.52 * \cos 20 * 0.15=69661.46 \mathrm{kgf}$

Lifting resistance $=$ Length of conveyor $*$ Total Weight $*$ $\sin \varnothing=28 * 17650.52 * \sin 20=169031.33 \mathrm{kgf}$

Considering $5 \%$ extra resistance $=0.05$

Terminal Resistance $=0.05(69659.41+169031.33)=$ $16934.53 \mathrm{kgf}$

Return Run Resistance $=28 * 46.13 * \cos 20 * 0.15=182.06$ kgf

Lift Resistance $=-28 * 46.13 * \sin 20=-441.76 \mathrm{kgf}$

Return Run Total $=182.06-441.76=-259.7 * 0.1=-25.97 \mathrm{kgf}$ Total Resistance $=$ Carrying Run side Resistance + Terminal Resistance + Total Return side Resistance

$=86570.09 \mathrm{kgf}$.

Power at Head Shaft $=($ Total Resistance $*$ Speed $) / 102=$ $212.18 \mathrm{~kW}$.

Drive Efficiency $=80 \%$.

Minimum power for drive $=0.8 * 212.18=169.74 \mathrm{~kW}$

\section{Material Selection}

When developing new products, it is necessary to consider a few mechanical attributes of the materials we wish to utilize. The fact is, material selection is very important because engineers have to plan for any potential consequences that certain materials may present.

Candidate Material Selection :- A group of materials is selected for the comparison of their properties and cost, feasible for the product. These materials selected are known as candidate materials. These candidate materials are selected using Ashby Chart.

Ashby Chart:- Almost all the properties of the materials can be known from this graph. This graph is very useful in comparing the properties of materials by finding the appropriate ratio between the respective materials.

There is a generic step-wise procedure for the selection of materials. These are also called the Quantitative methods for material selection. They are mainly categorized as :-
1. Cost per unit property method
2. Weighted properties method
3. Digital Logic method

\section{Material Selection Part :- Drive Shaft}

Function :-To support in combined loading.

Objective:-To increase fatigue strength.

Variables :-Density, Cross-sectional area.

Constraints :- Length, Force

Candidate materials selected for the drive shaft of slat conveyor :-

1) 4340 steel

2) Aluminum Alloy (2024-T6)

3) Titanium Alloy (Ti-6Al-4V)

Properties considered for material selection of slat conveyor

i) Elastic modulus

ii) Density iii) Tensile stress

iv) Yield stress

v) Working stress

vi) Factor of safety

Scaled Property Chart

\begin{tabular}{|c|c|c|c|c|}
\hline Property & $\begin{array}{c}\text { Positive } \\
\text { decision }\end{array}$ & $\begin{array}{c}\text { Weighing } \\
\text { ratio }\end{array}$ & Property & $\begin{array}{c}\text { Positive } \\
\text { decision }\end{array}$ \\
\hline $\begin{array}{c}\text { Elastic } \\
\text { modulus }\end{array}$ & 2 & 0.11 & $\begin{array}{c}\text { Elastic } \\
\text { modulus }\end{array}$ & 2 \\
\hline Density & 8 & 0.44 & Density & 8 \\
\hline $\begin{array}{c}\text { Tensile } \\
\text { stress }\end{array}$ & 5 & 0.277 & $\begin{array}{c}\text { Tensile } \\
\text { stress }\end{array}$ & 5 \\
\hline $\begin{array}{c}\text { Yield } \\
\text { stress }\end{array}$ & 3 & 0.166 & $\begin{array}{c}\text { Yield } \\
\text { stress }\end{array}$ & 3 \\
\hline
\end{tabular}

Numerical Values of the properties considered

\begin{tabular}{|c|c|c|c|c|c|c|}
\hline Materials & $\begin{array}{c}\text { Elastic } \\
\text { modulu } \\
\mathrm{s} \\
\left(\mathrm{N} / \mathrm{mm}^{2}\right. \\
)\end{array}$ & $\begin{array}{c}\text { Densit } \\
\mathrm{y} \\
\left(\mathrm{Kg} / \mathrm{m}^{3}\right. \\
)\end{array}$ & $\begin{array}{c}\text { Tensile } \\
\text { stress } \\
\left(\mathrm{N} / \mathrm{mm}^{2}\right. \\
)\end{array}$ & $\begin{array}{c}\text { Yield } \\
\text { stress } \\
\left(\mathrm{N} / \mathrm{mm}^{2}\right. \\
)\end{array}$ & $\begin{array}{c}\text { Workin } \\
\text { g stress } \\
\left(\mathrm{N} / \mathrm{mm}^{2}\right. \\
)\end{array}$ & $\begin{array}{c}\mathrm{FO} \\
\mathrm{S}\end{array}$ \\
\hline $\begin{array}{l}4340 \\
\text { Steel } \\
\end{array}$ & 210 & 7850 & 745 & 470 & 149 & 5 \\
\hline $\begin{array}{c}\text { Aluminu } \\
\text { m alloy } \\
(2024-T 6)\end{array}$ & 72.4 & 2780 & 427 & 345 & 85.4 & 5 \\
\hline $\begin{array}{c}\text { Titanium } \\
\text { Alloy } \\
\text { Ti-6Al-4 } \\
\text { V }\end{array}$ & 113 & 4430 & 950 & 880 & 190 & 5 \\
\hline
\end{tabular}

Relative Cost and Performance Index

\begin{tabular}{|c|c|c|c|}
\hline Material & Relative cost & $\begin{array}{c}\text { Performance } \\
\text { index }\end{array}$ & Final material \\
\hline 4340 steel & 9.2 & 85.26 & 784.39 \\
\hline $\begin{array}{c}\text { Aluminum } \\
\text { alloy } \\
\text { (2024-t6) }\end{array}$ & 6.2 & 38.24 & 237.10 \\
\hline $\begin{array}{c}\text { Titanium alloy } \\
\text { (Ti-6Al-4V) }\end{array}$ & 6.8 & 74.51 & 509.67 \\
\hline
\end{tabular}

Final selection of material by comparison of their respective performance index .For the final selection of the material the performance indices of the candidate material are compared. Performance index is given by $\boldsymbol{C}$.

\begin{tabular}{|c|c|c|c|c|}
\hline Materials & $\begin{array}{c}\text { Elastic } \\
\text { Modulus }\end{array}$ & Density & $\begin{array}{c}\text { Tensile } \\
\text { stress }\end{array}$ & $\begin{array}{c}\text { Yield } \\
\text { stress }\end{array}$ \\
\hline 4340 steel & 100 & 97.79 & 78.42 & 53.4 \\
\hline $\begin{array}{c}\text { Aluminum } \\
\text { alloy (2024-t6) }\end{array}$ & 34.47 & 34.63 & 44.94 & 39.2 \\
\hline & & & & \\
Titanium alloy \\
(Ti-6Al-4V)
\end{tabular}

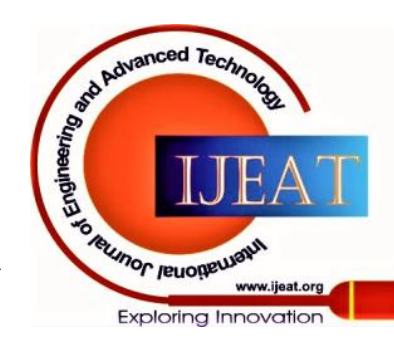




\section{Design and Optimisation of a Slat Conveyor for Airport Application}

The material with greater performance index is selected.

\subsection{Material Selection Part:- Slat}

Function :- To support in combined loading.

Objective :- To increase fatigue strength.

Variables :- Density, Cross sectional area.

Constraints :- Length, Force.

Candidate materials selected for the slat :-

1) AISI 1010

2) AISI 1020

3) AISI A36

4) AISI A516

5) IS 2062

Properties considered for material selection of slat conveyor :-
i. Elastic modulus
ii. Density
iii. Tensile stress
iv. Yield stress

Numerical values of the properties

\begin{tabular}{|c|c|c|c|c|}
\hline Material & $\begin{array}{c}\text { Elastic } \\
\text { modulus }\end{array}$ & Density & $\begin{array}{c}\text { Tensile } \\
\text { stress }\end{array}$ & $\begin{array}{c}\text { Yeild } \\
\text { strength }\end{array}$ \\
\hline AISI 1010 & 95.23 & 99.36 & 63.72 & 31.57 \\
\hline AISI 1020 & 95.2 & 100 & 82.35 & 61.55 \\
\hline AISI A36 & 95.23 & 99.36 & 78.43 & 43.85 \\
\hline AISI & 100 & 99.36 & 100 & 58.77 \\
\hline A516 2062 & 100 & 99.36 & 82.325 & 100 \\
\hline
\end{tabular}

Scaled Property Chart

\begin{tabular}{|c|c|c|c|c|}
\hline Material & $\begin{array}{c}\text { Elastic } \\
\text { modulus }\end{array}$ & Density & $\begin{array}{c}\text { Tensile } \\
\text { stress }\end{array}$ & $\begin{array}{c}\text { Yield } \\
\text { strength }\end{array}$ \\
\hline $\begin{array}{c}\text { AISI } \\
1010\end{array}$ & 200 & 7850 & 325 & 180 \\
\hline $\begin{array}{c}\text { AISI } \\
1020\end{array}$ & 200 & 7900 & 420 & 351 \\
\hline AISI A36 & 200 & 7850 & 400 & 250 \\
\hline $\begin{array}{c}\text { AISI } \\
\text { A516 }\end{array}$ & 210 & 7800 & 510 & 335 \\
\hline IS 2062 & 210 & 7850 & 420 & 570 \\
\hline
\end{tabular}

Digital Logic Method and Weighing Ratio

\begin{tabular}{|c|c|c|}
\hline Property & Positive decision & Weighing ratio \\
\hline Elastic modulus & 2 & 0.11 \\
\hline Density & 8 & 0.44 \\
\hline Tensile stress & 5 & 0.27 \\
\hline Yield strength & 3 & 0.16 \\
\hline
\end{tabular}

Final selection of material by comparison of their respective performance index.

\begin{tabular}{|c|c|c|c|}
\hline Material & Relative cost & $\begin{array}{c}\text { Performance } \\
\text { index }\end{array}$ & $\begin{array}{c}\text { Final } \\
\text { material }\end{array}$ \\
\hline AISI 1010 & 9.2 & 77.70 & 714.9 \\
\hline AISI 1020 & 6.2 & 88.16 & 546.62 \\
\hline AISI A36 & 6.8 & 83.84 & 570.12 \\
\hline AISI A516 & 6.4 & 92.56 & 592.42 \\
\hline IS 2062 & $\mathbf{1 . 9}$ & $\mathbf{9 4 . 8 1}$ & $\mathbf{1 8 0 . 1 5}$ \\
\hline
\end{tabular}

Thus in this case IS $\mathbf{2 0 6 2}$ is selected as it is having the highest performance index i.e94.81.

\subsection{Material Selection Part:- Baseframe}

Material Used: - Plain Carbon Steel

Properties of Plain Carbon Steel

\begin{tabular}{|c|c|}
\hline Properties & Values \\
\hline Density $\left(\mathrm{kg} / \mathrm{m}^{3}\right)$ & 7850 \\
\hline Yield strength $\left(\mathrm{N} / \mathrm{mm}^{2}\right)$ & 275 \\
\hline Young's modulus & 210000 \\
\hline
\end{tabular}

\section{CAD Modelling}

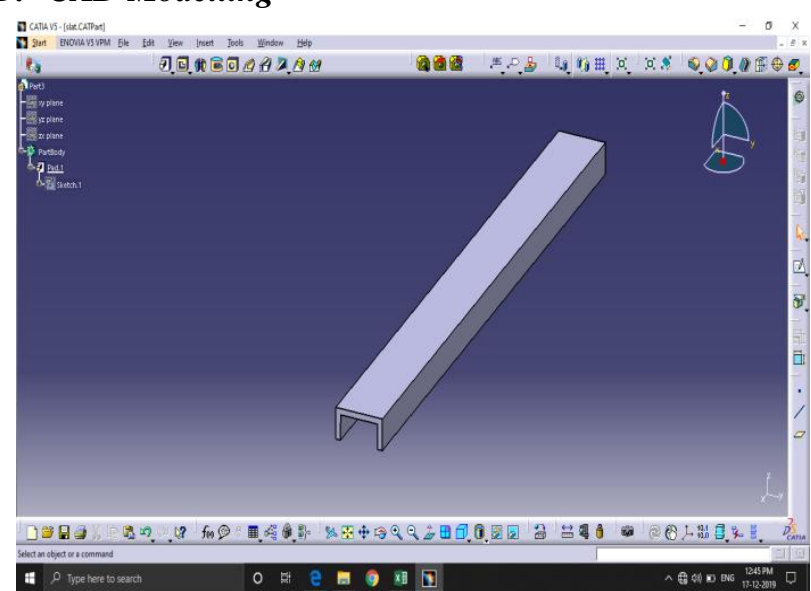

Fig 5.1: Slat Design

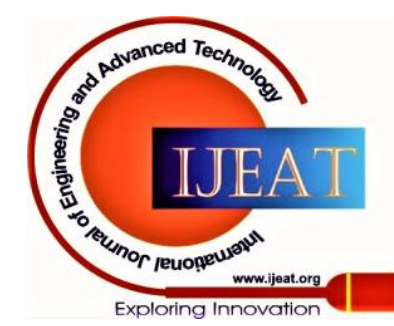




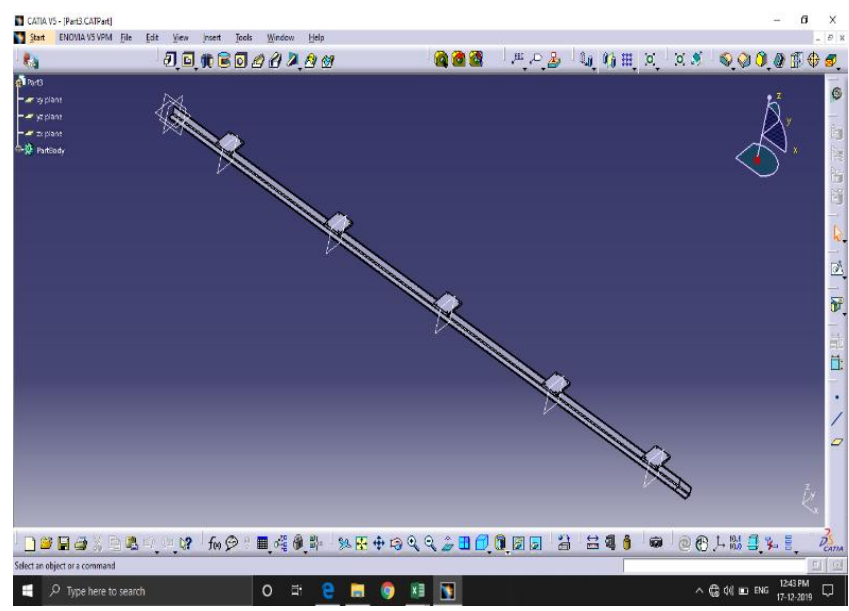

Fig 5.2: Conveyor Support

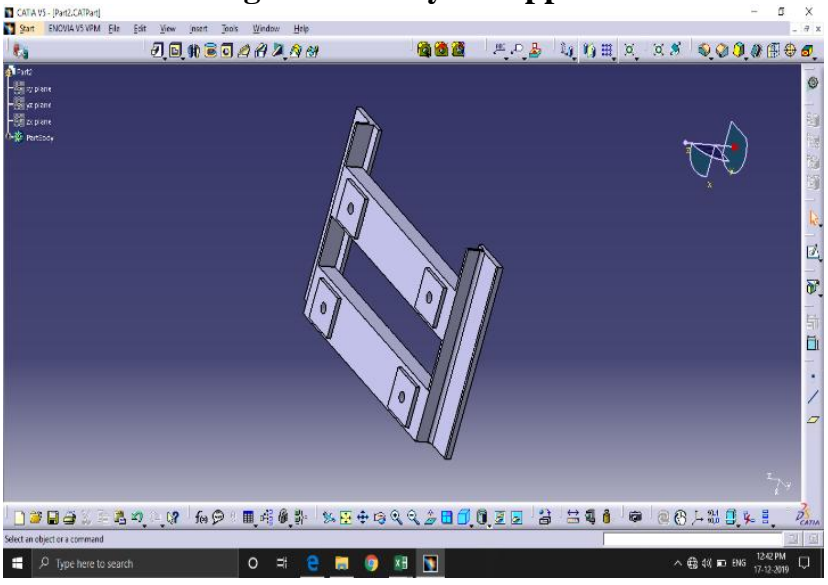

Fig 5.3: Fixed Support
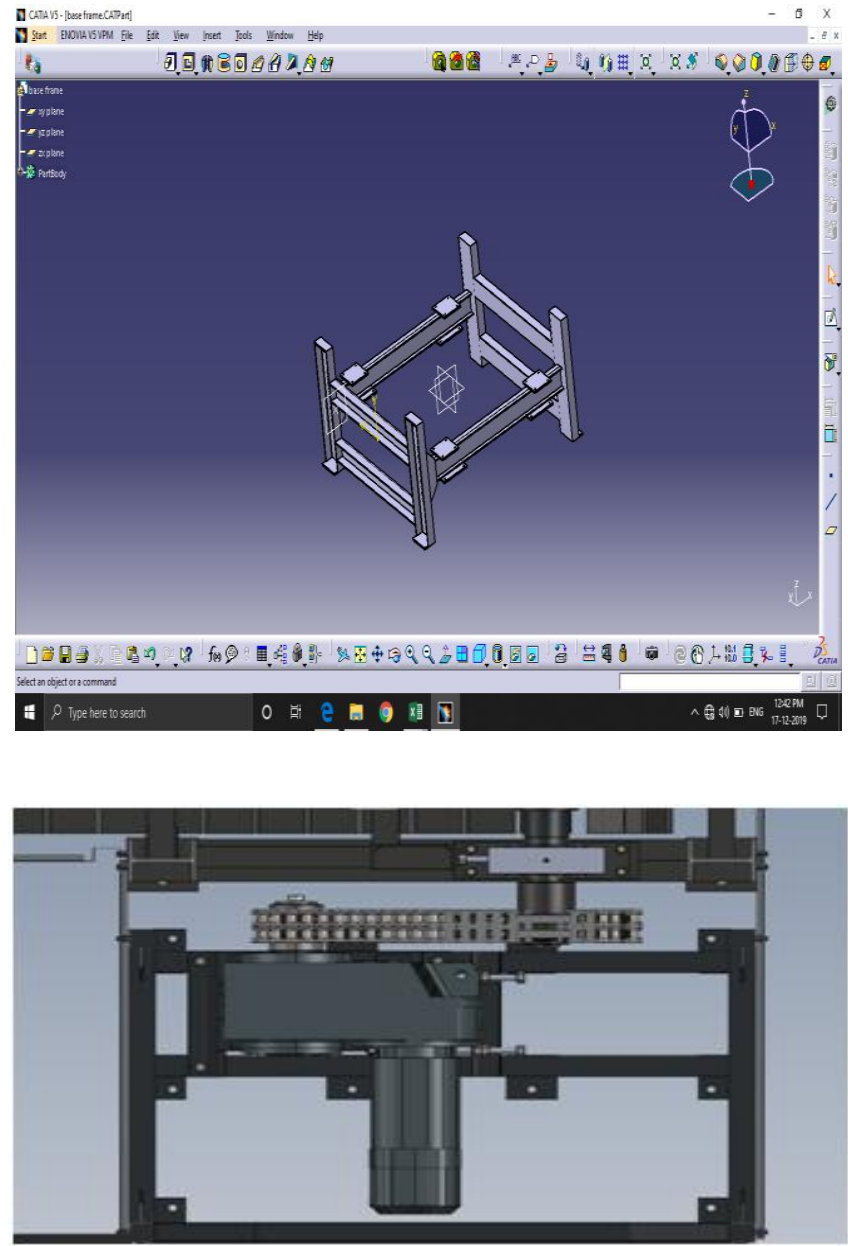

Fig 5.4: Chain Assembly (Top View)

Retrieval Number: 100.1/ijeat.E1132069520 DOI:10.35940/ijeat.E1132.1010120
Blue Eyes Intelligence Engineering and Sciences Publication

(C) Copyright: All rights reserved.
Published By:

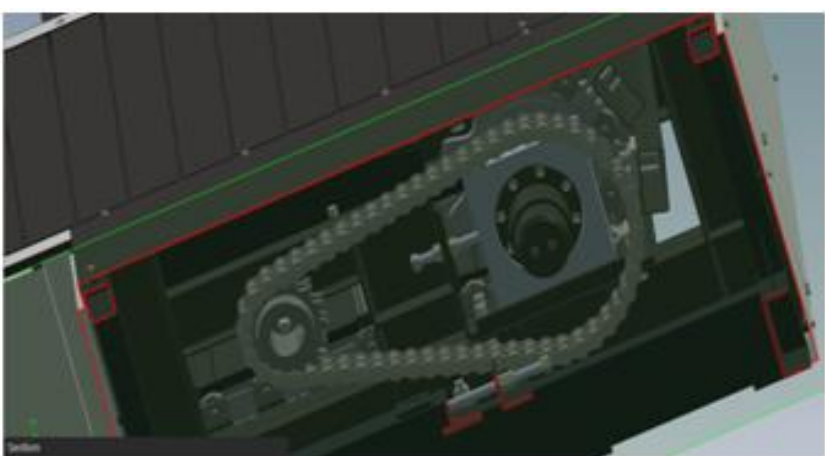

Fig 5.5: Chain Assembly (Top View)

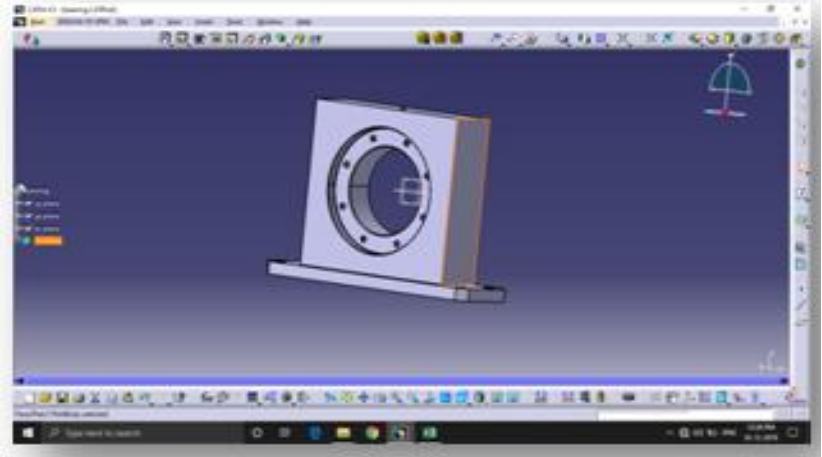

Fig 5.6: Bearing Support

\section{FINITE ELEMENT ANALYSIS}

\section{SHAFT}

\begin{tabular}{|c|l|}
\hline Model type: & $\begin{array}{l}\text { Linear Elastic } \\
\text { Isotropic }\end{array}$ \\
\hline $\begin{array}{c}\text { Default failure } \\
\text { criterion: }\end{array}$ & $\begin{array}{l}\text { Max von } \\
\text { Mises Stress }\end{array}$ \\
\hline Yield strength: & $\begin{array}{l}4.7 \mathrm{e}+08 \\
\mathrm{~N} / \mathrm{m}^{2}\end{array}$ \\
\hline Tensile strength: & $\begin{array}{l}7.45 \mathrm{e}+08 \mathrm{~N} / \\
\mathrm{m}^{2}\end{array}$ \\
\hline Elastic modulus: & $\begin{array}{l}2.1 \mathrm{e}+11 \mathrm{~N} / \\
\mathrm{m}^{2}\end{array}$ \\
\hline Poisson's ratio: & 0.28 \\
\hline Mass density: & $7850 \mathrm{~kg} / \mathrm{m}^{3}$ \\
\hline Shear modulus: & $\begin{array}{l}7.9 \mathrm{e}+10 \mathrm{~N} / \\
\mathrm{m}^{2}\end{array}$ \\
\hline $\begin{array}{l}\text { Thermal } \\
\text { expansion } \\
\text { coefficient: }\end{array}$ & $\begin{array}{l}1.3 \mathrm{e}-05 \\
/ \mathrm{Kelvin}\end{array}$ \\
\hline
\end{tabular}

Mesh Information

\begin{tabular}{|c|c|}
\hline Mesh Type & Solid Mesh \\
\hline Mesher Used & Blended Curvature based Mesh \\
\hline Jacobian Points & 4 points \\
\hline Maximum element Size & $45.8564 \mathrm{~mm}$ \\
\hline Minimum element size & $9.37128 \mathrm{~mm}$ \\
\hline Mesh Quality Plot & High \\
\hline
\end{tabular}

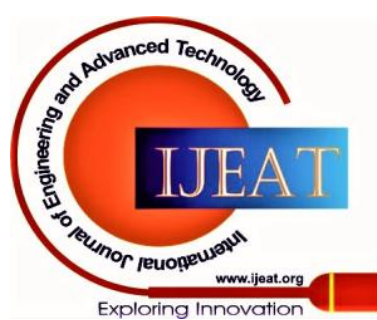




\section{Design and Optimisation of a Slat Conveyor for Airport Application}

Mesh Details

\begin{tabular}{|c|c|}
\hline Total Nodes & 37099 \\
\hline Total elements & 23519 \\
\hline Max. Aspect ratio & 33.389 \\
\hline
\end{tabular}

Resultant Forces

\begin{tabular}{|c|c|c|c|c|}
\hline Components & $\mathrm{x}$ & $\mathrm{y}$ & $\mathrm{z}$ & $\mathrm{F}_{\text {Resultant }}$ \\
\hline $\begin{array}{c}\text { Reaction } \\
\text { Force(N) }\end{array}$ & 1.38951 & 236628 & -1603.66 & 236633 \\
\hline $\begin{array}{c}\text { Reaction } \\
\text { moment(N-m) }\end{array}$ & 0 & 0 & 0 & 0 \\
\hline
\end{tabular}

Results

1) Stress

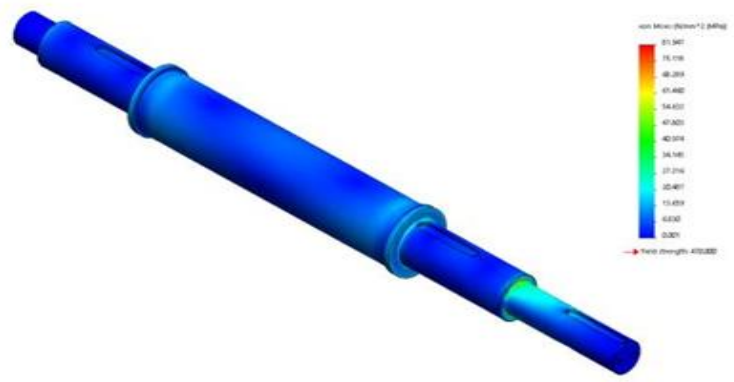

Max. Stress $=81.947 \mathrm{~N} / \mathrm{mm}^{2}$

2) Strain

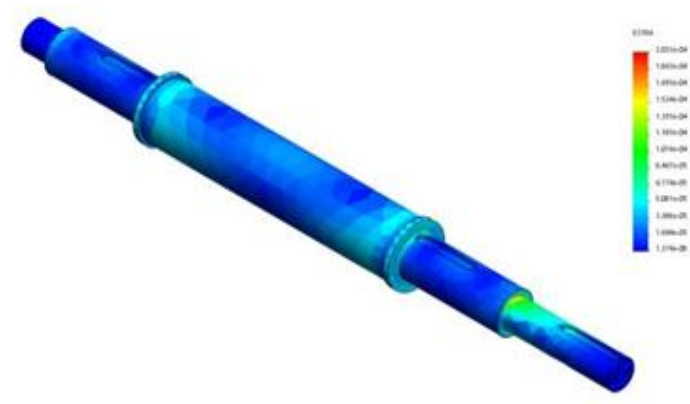

Max. Strain $=2.032 \mathrm{e}-04 \mathrm{~mm}$

3) Factor of Safety $=4.65 e+05$

Material 2 :2024-T3

\begin{tabular}{|c|c|}
\hline Model type: & Linear Elastic Isotropic \\
\hline $\begin{array}{c}\text { Default failure } \\
\text { criterion: }\end{array}$ & Max von Mises Stress \\
\hline Yield strength & $3.45 \mathrm{e}+08 \mathrm{~N} / \mathrm{m}^{2}$ \\
\hline Tensile strength & $4.85 \mathrm{e}+08 \mathrm{~N} / \mathrm{m}^{2}$ \\
\hline Elastic modulus & $7.24 \mathrm{e}+10 \mathrm{~N} / \mathrm{m}^{2}$ \\
\hline Poisson's ratio & 0.33 \\
\hline Mass density & $2780 \mathrm{~kg} / \mathrm{m}^{3}$ \\
\hline Shear modulus & $2.8 \mathrm{e}+10 \mathrm{~N} / \mathrm{m}^{2}$ \\
\hline $\begin{array}{c}\text { Thermal expansion } \\
\text { coefficient }\end{array}$ & $2.32 \mathrm{e}-05 / \mathrm{Kelvin}$ \\
\hline
\end{tabular}

Mesh Information

\begin{tabular}{|c|c|}
\hline Mesh Type & Solid Mesh \\
\hline Mesher Used & $\begin{array}{c}\text { Blended Curvature based } \\
\text { Mesh }\end{array}$ \\
\hline Jacobian Points & 4 points \\
\hline Maximum element Size & $45.8564 \mathrm{~mm}$ \\
\hline Minimum element size & $9.37128 \mathrm{~mm}$ \\
\hline Mesh Quality Plot & High \\
\hline
\end{tabular}

Mesh Details
\begin{tabular}{|c|c|}
\hline Total Nodes & 37099 \\
\hline Total elements & 23519 \\
\hline Max. Aspect ratio & 33.389 \\
\hline
\end{tabular}

Loads Applied

\begin{tabular}{|c|c|c|c|c|}
\hline \multicolumn{2}{|c|}{ Force } & \multicolumn{3}{|c|}{-22,000 N (Z-axis) } \\
\hline \multicolumn{2}{|c|}{ Torque } & \multicolumn{3}{|c|}{$-396 \mathrm{~N}-\mathrm{m}$} \\
\hline \multicolumn{5}{|c|}{ Forces(Resultant) } \\
\hline Components & $\mathrm{X}$ & $\mathrm{y}$ & $\mathrm{Z}$ & $F_{\text {Resultant }}$ \\
\hline $\mathrm{F}_{\text {Reaction }}(\mathrm{N})$ & -3.071 & 236625 & -1603.55 & 236631 \\
\hline $\begin{array}{c}\text { Reaction } \\
\text { moment(N-m) }\end{array}$ & 0 & 0 & 0 & 0 \\
\hline
\end{tabular}

Results

1) Stress

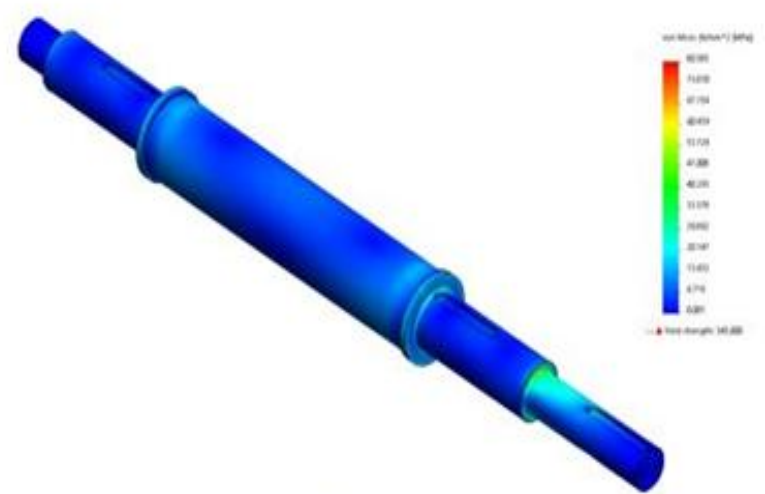

Max. Stress $=80.585 \mathrm{~N} / \mathrm{mm}^{2}$

2) Displacement

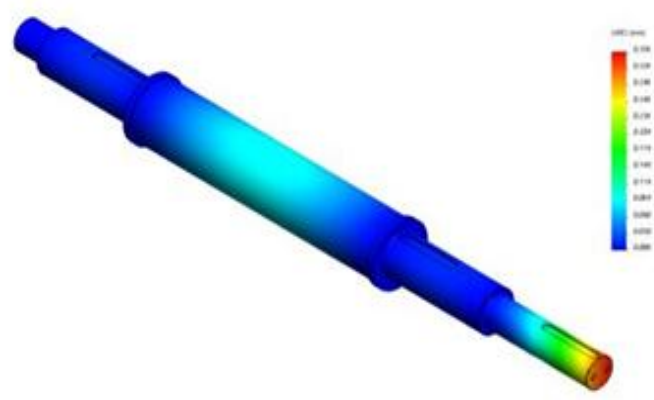

Max. Deformation $=0.358 \mathrm{~mm}$

3) Strain

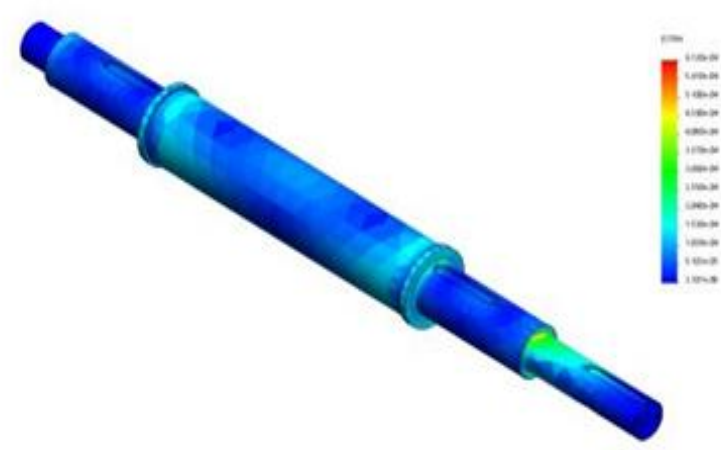

Max. Strain $=6.120 \mathrm{e}-04$

4) Factor of Safety $=3.51 e+05$.

Published By:

Blue Eyes Intelligence Engineering and Sciences Publication

(C) Copyright: All rights reserved.

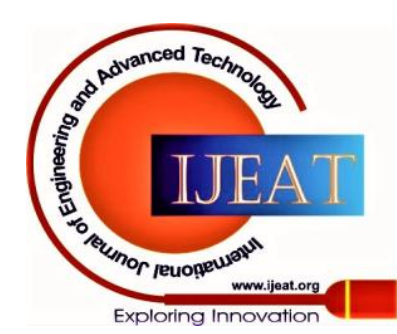


Material 3 : Ti-6Al-4VSolution treated and aged (SS)

\begin{tabular}{|c|c|}
\hline Model type & Linear Elastic Isotropic \\
\hline $\begin{array}{c}\text { Default failure } \\
\text { criterion }\end{array}$ & Max von Mises Stress \\
\hline Yield strength & $8.27371 \mathrm{e}+08 \mathrm{~N} / \mathrm{m}^{2}$ \\
\hline Tensile strength & $1.05 \mathrm{e}+09 \mathrm{~N} / \mathrm{m}^{2}$ \\
\hline Elastic modulus & $1.048 \mathrm{e}+11 \mathrm{~N} / \mathrm{m}^{2}$ \\
\hline Poisson's ratio & 0.31 \\
\hline Mass density & $4428.78 \mathrm{~kg} / \mathrm{m}^{3}$ \\
\hline Shear modulus & $4.10238 \mathrm{e}+10 \mathrm{~N} / \mathrm{m}^{2}$ \\
\hline
\end{tabular}

Mesh Information

\begin{tabular}{|c|c|}
\hline Mesh Type & Solid Mesh \\
\hline Mesher Used & $\begin{array}{c}\text { Blended Curvature based } \\
\text { Mesh }\end{array}$ \\
\hline Jacobian Points & 4 points \\
\hline Maximum element Size & $45.8564 \mathrm{~mm}$ \\
\hline Minimum element size & $9.37128 \mathrm{~mm}$ \\
\hline Mesh Quality Plot & High \\
\hline Mesh Details \\
\hline Total Nodes & 37099 \\
\hline Total elements & 23519 \\
\hline Max. Aspect ratio & 33.389 \\
\hline
\end{tabular}

Loads Applied

\begin{tabular}{|c|c|}
\hline Force & $-22,000 \mathrm{~N}$ (Z-axis) \\
\hline Torque & $-396 \mathrm{~N}-\mathrm{m}$ \\
\hline
\end{tabular}

Forces(Resultant)

\begin{tabular}{|c|c|c|c|c|}
\hline Components & $\mathrm{x}$ & $\mathrm{y}$ & $\mathrm{z}$ & $\mathrm{F}_{\text {Resultant }}$ \\
\hline $\mathrm{F}_{\text {Reaction }}(\mathrm{N})$ & 4.782 & 236626 & -1601.48 & 236631 \\
\hline $\begin{array}{c}\text { Reaction } \\
\text { moment }(\mathrm{N}-\mathrm{m})\end{array}$ & 0 & 0 & 0 & 0 \\
\hline
\end{tabular}

Results

1) Stress

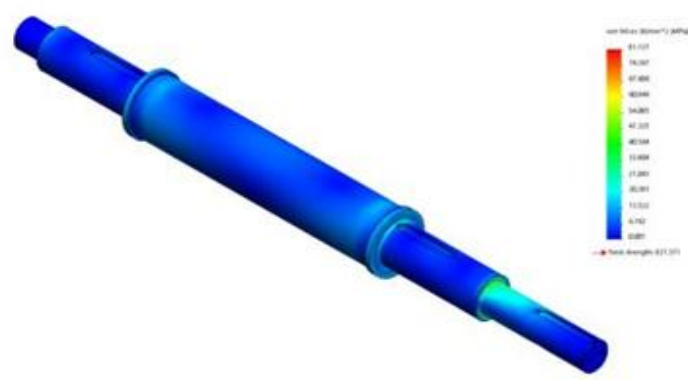

Max. Stress $=81.127 \mathrm{~N} / \mathrm{mm}^{2}$

2) Displacement

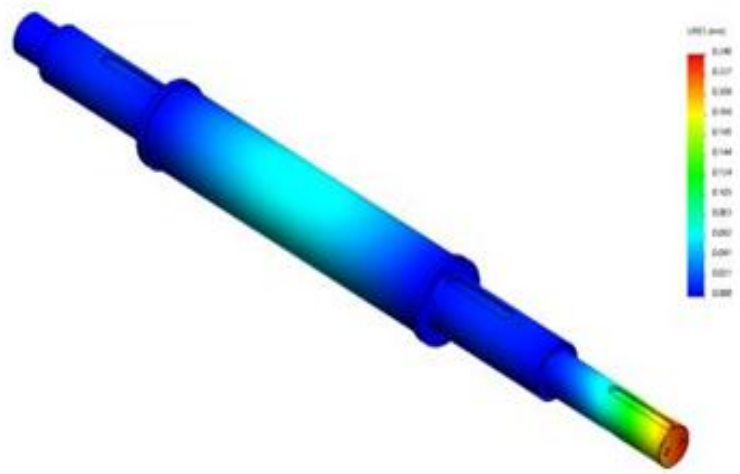

Max. Displacement $=0.248 \mathrm{~mm}$.
3) Strain

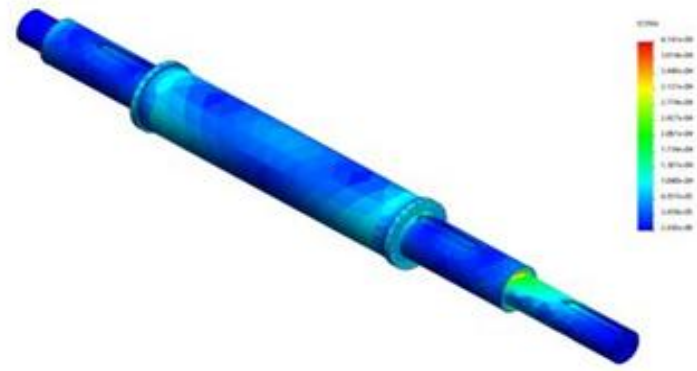

Max. Strain $=4.161 \mathrm{e}-04$

4) Factor of Safety $=\mathbf{8 . 3 4 e + 0 5}$.

SLAT

Dimension $1: \mathbf{7 5 * 4 0 * 3}$

Material Used: - Plain Carbon Steel

\begin{tabular}{|c|c|}
\hline Model type & Linear Elastic Isotropic \\
\hline Default failure criterion & Unknown \\
\hline Yield strength & $2.20594 \mathrm{e}+08 \mathrm{~N} / \mathrm{m}^{2}$ \\
\hline Tensile strength & $3.99826 \mathrm{e}+08 \mathrm{~N} / \mathrm{m}^{2}$ \\
\hline Elastic modulus & $2.1 \mathrm{e}+11 \mathrm{~N} / \mathrm{m}^{2}$ \\
\hline Poisson's ratio & 0.28 \\
\hline Mass density & $7800 \mathrm{~kg} / \mathrm{m}^{3}$ \\
\hline Shear modulus & $7.9 \mathrm{e}+10 \mathrm{~N} / \mathrm{m}^{2}$ \\
\hline $\begin{array}{c}\text { Thermal expansion } \\
\text { coefficient }\end{array}$ & $1.3 \mathrm{e}-05 /$ Kelvin \\
\hline
\end{tabular}

Mesh Information

\begin{tabular}{|c|c|}
\hline Mesh Type & Solid Mesh \\
\hline Mesher Used & Standard Mesh \\
\hline Jacobian Points & 4 points \\
\hline Element Size & $18.8261 \mathrm{~mm}$ \\
\hline Tolerance & $0.941303 \mathrm{~mm}$ \\
\hline Mesh Quality Plot & High \\
\hline
\end{tabular}

Mesh Details

\begin{tabular}{|c|c|}
\hline Total Nodes & 27159 \\
\hline Total elements & 15209 \\
\hline Max. Aspect ratio & 41.263 \\
\hline
\end{tabular}

Loads Applied

\begin{tabular}{|c|c|}
\hline Force & $5400 \mathrm{~N}(\mathrm{Normal})$ \\
\hline Torque & $0 \mathrm{~N}-\mathrm{m}$ \\
\hline
\end{tabular}

Forces(Resultant)

Forces(Resultant)
\begin{tabular}{|c|c|c|c|c|}
\hline Components & $\mathrm{x}$ & $\mathrm{y}$ & $\mathrm{z}$ & $\mathrm{F}_{\text {Resultant }}$ \\
\hline $\mathrm{F}_{\text {Reaction }}(\mathrm{N})$ & $\begin{array}{c}-1.6 \\
856\end{array}$ & 5397.34 & 1.73 & 5937.34 \\
\hline $\begin{array}{c}\text { Reaction } \\
\text { moment }(\mathrm{N}-\mathrm{m})\end{array}$ & 0 & 0 & 0 & 0 \\
\hline
\end{tabular}

Results

1) Stress

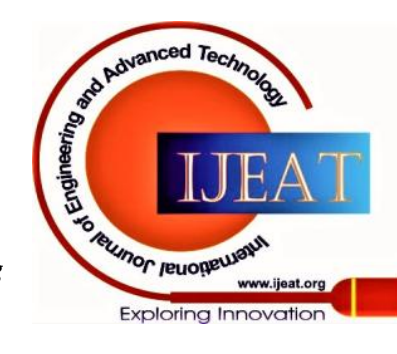




\section{Design and Optimisation of a Slat Conveyor for Airport Application}

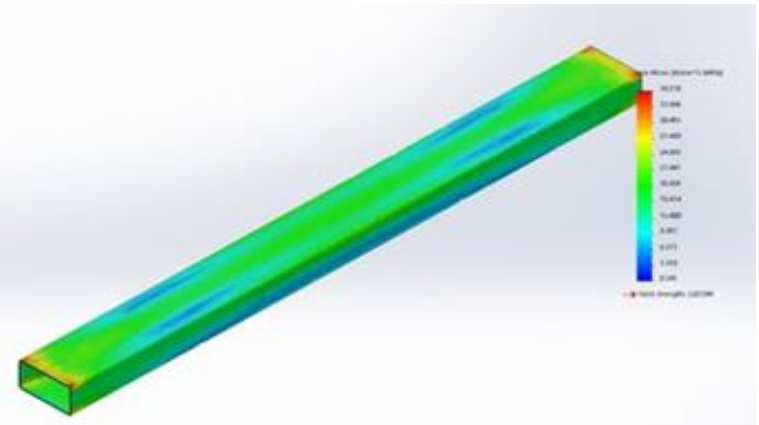

Max. Stress $=36.510 \mathrm{~N} / \mathrm{mm}^{2}$

2) Displacement

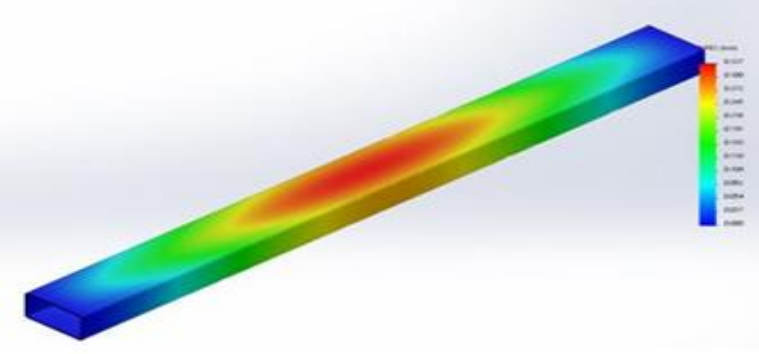

Max. Displacement $=0.327 \mathrm{~mm}$

3) Strain

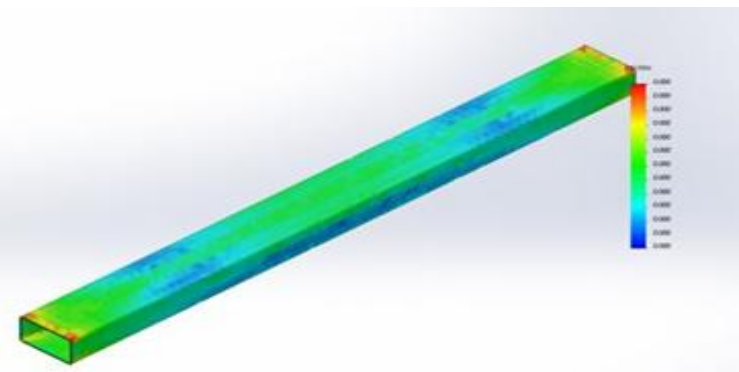

Max. Srain: 0.

Dimension $2: \mathbf{7 5} * \mathbf{4 0} * \mathbf{5}$

Material Used: - Plain Carbon Steel

\begin{tabular}{|c|c|}
\hline Model type & Linear Elastic Isotropic \\
\hline Default failure criterion & Unknown \\
\hline Yield strength & $2.20594 \mathrm{e}+08 \mathrm{~N} / \mathrm{m}^{2}$ \\
\hline Tensile strength & $3.99826 \mathrm{e}+08 \mathrm{~N} / \mathrm{m}^{2}$ \\
\hline Elastic modulus & $2.1 \mathrm{e}+11 \mathrm{~N} / \mathrm{m}^{2}$ \\
\hline Poisson's ratio & 0.28 \\
\hline Mass density & $7800 \mathrm{~kg} / \mathrm{m}^{3}$ \\
\hline Shear modulus & $7.9 \mathrm{e}+10 \mathrm{~N} / \mathrm{m}^{2}$ \\
\hline $\begin{array}{c}\text { Thermal expansion } \\
\text { coefficient }\end{array}$ & $1.3 \mathrm{e}-05 / \mathrm{Kelvin}$ \\
\hline
\end{tabular}

Mesh Information

\begin{tabular}{|c|c|}
\hline Mesh Type & Solid Mesh \\
\hline Mesher Used & Standard Mesh \\
\hline Jacobian Points & 4 points \\
\hline Element Size & $18.8261 \mathrm{~mm}$ \\
\hline Tolerance & $0.941303 \mathrm{~mm}$ \\
\hline Mesh Quality Plot & High \\
\hline \multicolumn{2}{|c|}{ Mesh Details } \\
\hline Total Nodes & 27159 \\
\hline Total elements & 15209 \\
\hline Max. Aspect ratio & 41.263 \\
\hline
\end{tabular}

Loads Applied

\begin{tabular}{|c|c|c|c|c|}
\hline \multicolumn{2}{|c|}{ Force } & \multicolumn{3}{c|}{$5400 \mathrm{~N}($ Normal) } \\
\hline \multicolumn{2}{|c|}{ Torque } & \multicolumn{3}{c|}{$0 \mathrm{~N}-\mathrm{m}$} \\
\hline \\
Forces(Resultant) \\
\hline $\begin{array}{c}\text { Components } \\
\text { Reaction } \\
\text { Force(N) }\end{array}$ & -0.1206 & 5399.65 & 0.066 & 5399.64 \\
\hline $\begin{array}{c}\text { Reaction } \\
\text { moment(N-m) }\end{array}$ & 0 & 0 & 0 & 0 \\
\hline
\end{tabular}

Results

1) Stress

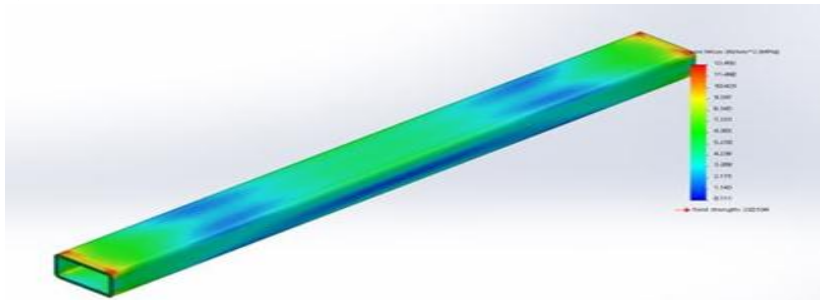

Max. Stress $=12.492 \mathrm{~N} / \mathrm{mm}^{2}$

2) Displacement

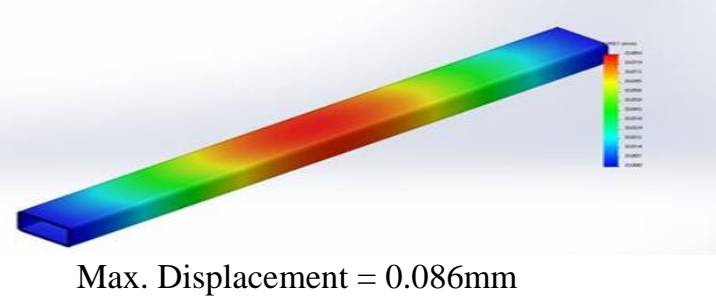

3) Strain

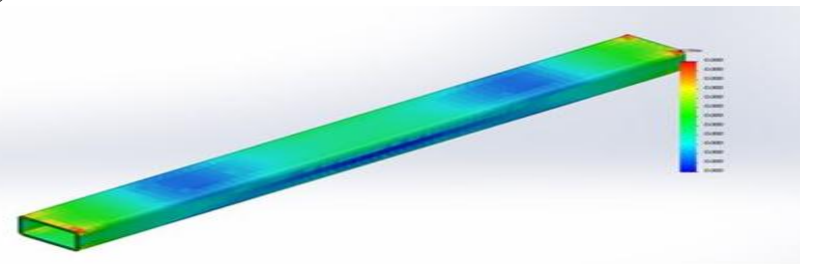

BASE FRAME

Max. Strain $=0$.

Material Used :- Plain Carbon Steel

Material Properties

\begin{tabular}{|c|c|}
\hline Model type & Linear Elastic Isotropic \\
\hline Default failure criterion & Unknown \\
\hline Yield strength & $2.20594 \mathrm{e}+08 \mathrm{~N} / \mathrm{m}^{2}$ \\
\hline Tensile strength & $3.99826 \mathrm{e}+08 \mathrm{~N} / \mathrm{m}^{2}$ \\
\hline Elastic modulus & $2.1 \mathrm{e}+11 \mathrm{~N} / \mathrm{m}^{2}$ \\
\hline Poisson's ratio & 0.28 \\
\hline Mass density & $7800 \mathrm{~kg} / \mathrm{m}^{3}$ \\
\hline Shear modulus & $7.9 \mathrm{e}+10 \mathrm{~N} / \mathrm{m}^{2}$ \\
\hline $\begin{array}{c}\text { Thermal expansion } \\
\text { coefficient }\end{array}$ & $1.3 \mathrm{e}-05 /$ Kelvin \\
\hline
\end{tabular}

Mesh Information

\begin{tabular}{|c|c|}
\hline Mesh Type & Solid Mesh \\
\hline Mesher Used & Standard Mesh \\
\hline Jacobian Points & 4 points \\
\hline Element Size & $47.9735 \mathrm{~mm}$ \\
\hline Tolerance & $0.5 \mathrm{~mm}$ \\
\hline Mesh Quality Plot & Draft Quality Mesh \\
\hline
\end{tabular}

Published By:

Blue Eyes Intelligence Engineering

and Sciences Publication

(C) Copyright: All rights reserved. 
Mesh Details

\begin{tabular}{|c|c|}
\hline Total Nodes & 7314 \\
\hline Total elements & 21813 \\
\hline Max. Aspect ratio & 99.719 \\
\hline Loads Applied \\
\hline Force & 2156 N(Normal) \\
\hline Torque & 0 N-m \\
\hline
\end{tabular}

Forces(Resultant)

\begin{tabular}{|c|c|c|c|c|}
\hline Components & $\mathrm{x}$ & $\mathrm{y}$ & $\mathrm{z}$ & $\mathrm{F}_{\text {Resultant }}$ \\
\hline $\begin{array}{c}\text { Reaction } \\
\text { Force(N) }\end{array}$ & -4.7024 & 17246.7 & -0.74 & $\begin{array}{c}17246 . \\
77\end{array}$ \\
\hline $\begin{array}{c}\text { Reaction } \\
\text { moment(N-m } \\
\text { ) }\end{array}$ & 0 & 0 & 0 & 0 \\
\hline
\end{tabular}

Results

1) Stress

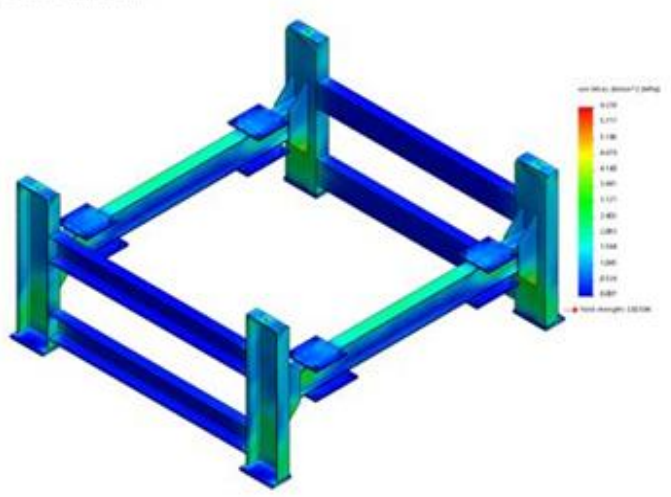

Max. Stress $=6.236 \mathrm{~N} / \mathrm{mm}^{2}$

2) Displacement

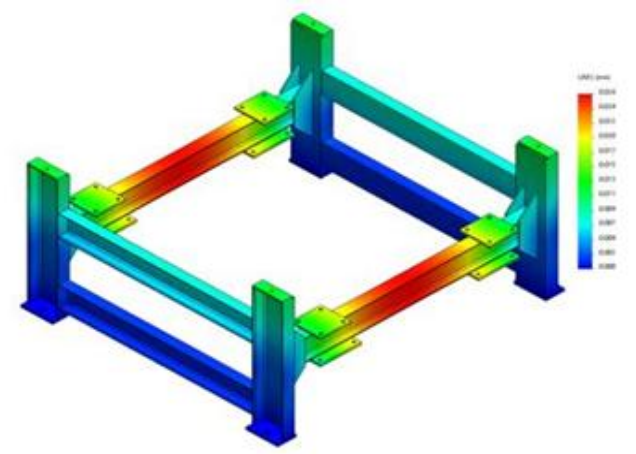

Max. Displacement $=0.026 \mathrm{~mm}$

3) Strain

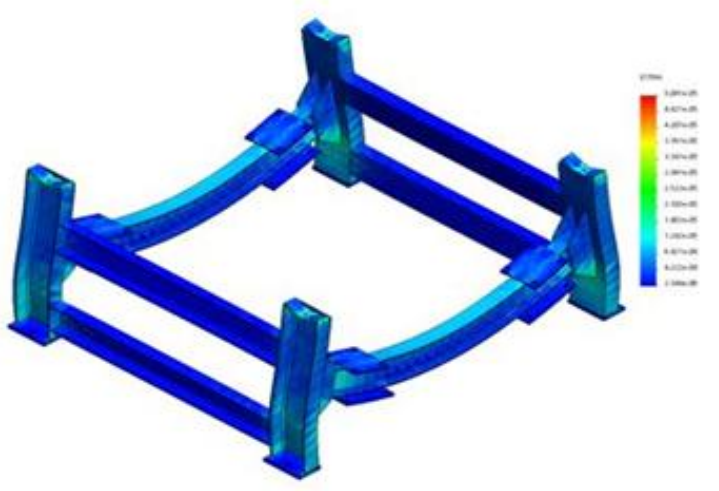

Max. Strain $=5.041 \mathrm{e}-05$

4) Factor of Safety

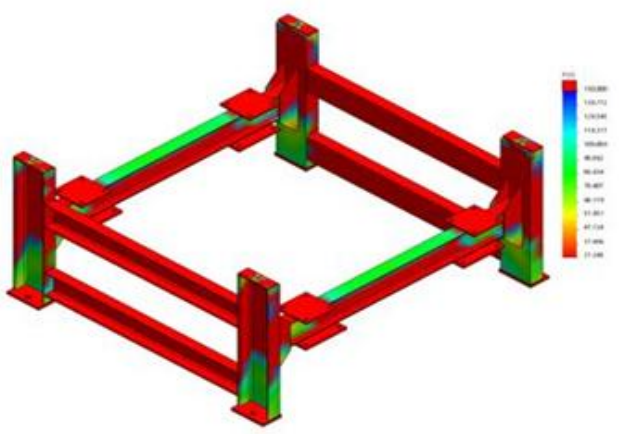

Max. Value $=31565.008$

\section{CONCLUSION}

After observing the analysis of the above components the objective of the research has been fulfilled. The material for the components is selected according to the Industrial standards like Ashby and CEMA. The analysis was done for each iteration to optimize the design and also the cost as well as the weight of the components. Through this research we have designed the shaft that will work efficiently with improved life cycle.

\section{REFERENCES}

1. Design and Analysis of a Conveyor. (ICIIIME 2017) ISSN : 2321-8169

2. Ketten Handbuch, Iwis- High performance chains. Sprocket and pinions for precision roller.

3. Chain simplex, recommended by NU-TECH, Page-11.

4. V.B. Bhandari "Design of Machine Elements" book.

\section{AUTHORS PROFILE}

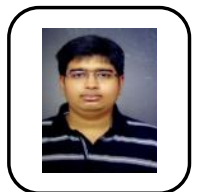

Vatsal Singh is a research scholar currently studying as a Final year student from MIT Academy of Engineering in the Mechanical Engineering. He has been an active member of Autosport's club. He has also participated in events like BAJA SAEINDIA and ISIE-IKR.

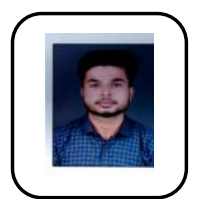

Sanskar Joshi is a research scholar currently studying as a Final year student from MIT Academy of Engineering in the Mechanical Engineering. He has been an active member of Autosport's club. He has also participated in events like BAJA SAEINDIA.

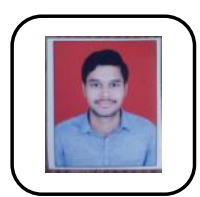

Siddheshwar Wahude is currently studying as a Final year student from MIT Academy of Engineering in the Mechanical Engineering.

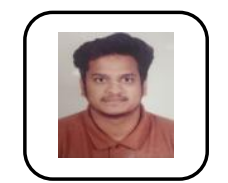

Sahil Shaikh is a research scholar currently studying as a Final year student from MIT Academy of Engineering in the Mechanical Engineering.

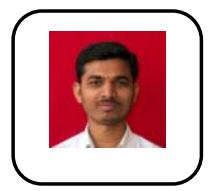

Dilip Panchal (Guide), Associate Professor, MIT Academy of Engineering.

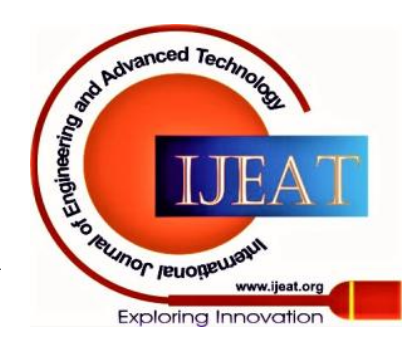

